\title{
On exceptional sets in the metric Poissonian pair correlations problem
}

\author{
Thomas Lachmann $^{1}$ (D) Niclas Technau ${ }^{1}$
}

Received: 7 February 2018 / Accepted: 30 May 2018 / Published online: 13 June 2018

(C) The Author(s) 2018

\begin{abstract}
Let $\left(a_{n}\right)_{n}$ be a strictly increasing sequence of positive integers. Recent works uncovered a close connection between the additive energy $E\left(A_{N}\right)$ of the cut-offs $A_{N}=\left\{a_{n}: n \leq N\right\}$, and $\left(a_{n}\right)_{n}$ possessing metric Poissonian pair correlations which is a metric version of a uniform distribution property of "second order". Firstly, the present article makes progress on a conjecture of Aichinger, Aistleitner, and Larcher; by sharpening a theorem of Bourgain which states that the set of $\alpha \in[0,1]$ satisfying that $\left(\left\langle\alpha a_{n}\right\rangle\right)_{n}$ with $E\left(A_{N}\right)=\Omega\left(N^{3}\right)$ does not have Poissonian pair correlations has positive Lebesgue measure. Secondly, we construct sequences with high additive energy which do not have metric Poissonian pair correlations, in a strong sense, and provide Hausdorff dimension estimates.
\end{abstract}

Keywords Poissonian pair correlations · Additive energy · Diophantine approximation - Metric number theory

Communicated by A. Constantin.

During the submission process of this manuscript, said conjecture has been proven by Larcher and Stockinger [10].

Thomas Lachmann is supported by the Austrian Science Fund (FWF): Y-901. Niclas Technau is supported by the Austrian Science Fund (FWF) Projects: W1230, and (partially) by Y-901.

$\bowtie$ Thomas Lachmann

lachmann@math.tugraz.at

Niclas Technau

technau@math.tugraz.at

15010 Institut für Analysis und Zahlentheorie, Steyrergasse 30/II, 8010 Graz, Austria 
Mathematics Subject Classification Primary 11B30; Secondary 11B25 - 11K06 • $11 \mathrm{~K} 60$

\section{Introduction}

The theory of uniform distribution modulo 1 dates back, at least, to the seminal paper [23] of Weyl who showed that for any fixed $\alpha \in \mathbb{R} \backslash \mathbb{Q}$ and integer $d \geq 1$ the sequences $\left(\left\langle\alpha n^{d}\right\rangle\right)_{n}$ are uniformly distributed modulo 1 where $\langle x\rangle$ denotes the fractional part of $x \in \mathbb{R}$. However, in recent years various authors $[2,4,5,9,11,14$ $18,21,22]$ have been investigating a more subtle distribution property of such sequences - namely, whether the asymptotic distribution of the pair correlations has a property which is called Poissonian, and defined as follows.

Definition Let $\|\cdot\|$ denote the distance to the nearest integer. A sequence $\left(\theta_{n}\right)_{n}$ in $[0,1]$ is said to have (asymptotically) Poissonian pair correlations, for shorthand notation PPC, if for each $s \geq 0$ the pair correlation function ${ }^{1}$

$$
R_{2}\left([-s, s],\left(\theta_{n}\right)_{n}, N\right):=\frac{1}{N} \#\left\{1 \leq i \neq j \leq N:\left\|\theta_{i}-\theta_{j}\right\| \leq \frac{s}{N}\right\}
$$

tends to $2 s$ as $N \rightarrow \infty$. Moreover, let $\left(a_{n}\right)_{n}$ denote a strictly increasing sequence of positive integers. If no confusion can arise, we write

$$
R([-s, s], \alpha, N):=R_{2}\left([-s, s],\left(\alpha a_{n}\right)_{n}, N\right)
$$

and say that a sequence $\left(a_{n}\right)_{n}$ has metric Poissonian pair correlations if $\left(\alpha a_{n}\right)_{n}$ has PPC for almost all $\alpha \in[0,1]$ where almost all throughout this article is meant with respect to the Lebesgue measure.

It is known that if a sequence $\left(\theta_{n}\right)_{n}$ has PPC, then it is uniformly distributed modulo 1 , cf. $[3,13,19]$. Yet, the sequences $\left(\left\langle\alpha n^{d}\right\rangle\right)_{n}$ do not have PPC for any $\alpha \in \mathbb{R}$ if $d=1$. For $d \geq 2$, Rudnick and Sarnak [16] proved that $\left(n^{d}\right)_{n}$ has metric Poissonian pair correlations (metric PPC). A result of Aistleitner et al. [2], who used a Fourier analytic approach combined with a bound on GCD sums of Bondarenko and Seip [6], uncovered the connection of the metric PPC property of $\left(a_{n}\right)_{n}$ with its combinatoric properties. For stating it, we introduce some notation. Let $\left(a_{n}\right)_{n}$ denote throughout this article a strictly increasing sequence of positive integers, and abbreviate the set of the first $N$ elements of $\left(a_{n}\right)_{n}$ by $A_{N}$. Moreover, define the additive energy $E(I)$ of a finite set of integers $I$ via

$$
E(I):=\#\left\{(a, b, c, d) \in I^{4}: a+b=c+d\right\},
$$

and note that $(\# I)^{2} \leq E(I) \leq(\# I)^{3}$ where $\# S$ denotes the cardinality of a set $S$. In the following, let $\mathcal{O}$ and $o$ denote the Landau symbols/O-notation, and $\ll$ or $\gg$ the

\footnotetext{
1 The subscript 2 in $R_{2}$ indicates that relations of second order, i.e. pair correlations, are counted.
} 
Vinogradov symbols. The dependence of an implied constant in one of these symbols will be indicated by mentioning this parameter in a subscript.

Now, a main finding of [2] can be stated as the implication that if the truncations $A_{N}$ satisfy

$$
E\left(A_{N}\right)=\mathcal{O}\left(N^{3-\varepsilon}\right)
$$

for some fixed $\varepsilon>0$, then $\left(a_{n}\right)_{n}$ has metric PPC. Roughly speaking, a set $I$ has large additive energy if and only if it contains a "large" arithmetic progression like structure. Indeed, if $\left(a_{n}\right)_{n}$ is a geometric progression or of the form $\left(n^{d}\right)_{n}$ for $d \geq 2$, then (2) is satisfied.

Recently, Bloom, Chow, Gafni, Walker relaxed—provided that, roughly speaking, the density of the sequence does not decay faster than $1 /(\log N)^{2}$ — the power saving bound (2) for detecting the metric PPC property of $\left(a_{n}\right)_{n}$ significantly:

Theorem A [4] If there exists an $\varepsilon>0$ such that

$$
E\left(A_{N}\right) \ll_{\varepsilon} \frac{N^{3}}{(\log N)^{2+\varepsilon}} \text { and } \frac{1}{N} \#\left(A_{N} \cap\{1, \ldots, N\}\right) \gg_{\varepsilon} \frac{1}{(\log N)^{2+2 \varepsilon}}
$$

hold, then $\left(a_{n}\right)_{n}$ has metric PPC.

In accordance with probabilistic considerations, cf. [4, Thm. 1.5], the above result could be seen as a sign of Khintchine-type law underpinning the characterization of the metric PPC property of $\left(a_{n}\right)_{n}$. Indeed, the following basic question about the nature of the connection between additive energy and the metric PPC property was raised in [4]:

Fundamental Question [4] Is it true that if $E\left(A_{N}\right) \sim N^{3} \psi(N)$ for some weakly decreasing function $\psi: \mathbb{N} \rightarrow[0,1]$, then $\left(a_{n}\right)_{n}$ has metric PPC if and only if $\sum_{N \geq 1} \psi(N) / N$ converges?

Remark This question will be answered in the negative in a forthcoming note of Aistleitner and the authors.

Regarding the optimal bound for $E\left(A_{N}\right)$ to ensure the metric PPC property of $\left(a_{n}\right)_{n}$, the following two questions were raised in [2]. For stating those, we use the convention that $f=\Omega(g)$ means for $f, g: \mathbb{N} \rightarrow \mathbb{R}$ there is a constant $c>0$ such that $g(n)>c f(n)$ holds for infinitely many $n$.

Question 1 Is it possible for $\left(a_{n}\right)_{n}$ with $E\left(A_{N}\right)=\Omega\left(N^{3}\right)$ to have metric PPC?

Question 2 Do all $\left(a_{n}\right)_{n}$ with $E\left(A_{N}\right)=o\left(N^{3}\right)$ have metric PPC?

Both questions were answered in the negative by Bourgain whose proofs can be found in [2] as an appendix, without giving an estimate on the measure of the set that was used to answer Question 1, and without a quantitative bound on $E\left(A_{N}\right)$ appearing in the negation of Question 2. However, a quantitative analysis, as noted in [22], shows that the sequence Bourgain constructed for Question 2 satisfies

$$
E\left(A_{N}\right)=\mathcal{O}_{\varepsilon}\left(\frac{N^{3}}{(\log \log N)^{\frac{1}{4}+\varepsilon}}\right)
$$


for any fixed $\varepsilon>0$. Moreover, Nair posed the problem ${ }^{2}$ whether the sequence of prime numbers $\left(p_{n}\right)_{n}$, ordered by increasing value, has metric PPC. Recently, Walker [22] answered this question in the negative by showing that there is a constant $c>0$ satisfying that for almost every $\alpha \in[0,1]$ the inequality $R([-s, s], \alpha, N)>c$ holds for infinitely many $N$. Thereby he gave a significantly better bound than (3) for the additive energy $E\left(A_{n}\right)$ for a sequence $\left(a_{n}\right)_{n}$ not having metric PPC — since the additive energy of the truncations of $\left(p_{n}\right)_{n}$ is in $\Theta\left((\log N)^{-1} N^{3}\right)$ where $f=\Theta(g)$, for functions $f, g$, means that both $f=\mathcal{O}(g)$ and $g=\mathcal{O}(f)$ holds.

For a given sequence $\left(a_{n}\right)_{n}$, we denote by NPPC $\left(\left(a_{n}\right)_{n}\right)$ the "exceptional" set of all $\alpha \in(0,1)$ such that $\left(\left\langle\alpha a_{n}\right\rangle\right)_{n}$ does not have PPC.

Theorem B [2] If $E\left(A_{N}\right)=\Omega\left(N^{3}\right)$, then NPPC $\left(\left(a_{n}\right)_{n}\right)$ has positive Lebesgue measure.

We prove the following sharpening.

Theorem 1 If $E\left(A_{N}\right)=\Omega\left(N^{3}\right)$, then NPPC $\left(\left(a_{n}\right)_{n}\right)$ has full Lebesgue measure.

Some remarks are in order.

Remark (a) $\left(a_{n}\right)_{n}$ is called quasi-arithmetic of degree one, cf. [1, Def. 1], if infinitely often at least a constant proportion of elements of $A_{N}$ is contained in some arithmetic progression of length $\ll N$. Any such sequence obviously satisfies $E\left(A_{N}\right)=\Omega\left(N^{3}\right)$. Theorem 1 improves upon a recent result of Aichinger, Aistleitner, and Larcher [1, Thm. 3] who showed that NPPC $\left(\left(a_{n}\right)_{n}\right)$ has full Lebesgue measure, if $\left(a_{n}\right)_{n}$ is quasi-arithmetic of degree one.

(b) Recently, Larcher [12, Thm. 1] sharpened this result to $\operatorname{NPPC}\left(\left(a_{n}\right)_{n}\right)=(0,1)$, and subsequently Larcher and Stockinger [10, Thm. 1] extended this to quasiarithmetic sequences of any degree $d \geq 1-$ which due to Freiman's theorem (cf. [12, text above Def. 2]) implies that if $E\left(A_{N}\right)=\Omega\left(N^{3}\right)$, then $\operatorname{NPPC}\left(\left(a_{n}\right)_{n}\right)=$ $(0,1)$.

For stating our second main theorem, we denote by $\mathbb{R}_{>x}$ the set of real numbers exceeding a given $x \in \mathbb{R}$.

Theorem 2 Let $f: \mathbb{R}_{>0} \rightarrow \mathbb{R}_{>2}$ be a function increasing monotonically to $\infty$, and satisfying $f(x)=\mathcal{O}\left(x^{1 / 3}(\log x)^{-7 / 3}\right)$. Then, there is a strictly increasing sequence $\left(a_{n}\right)_{n}$ of positive integers with $E\left(A_{N}\right)=\Theta\left(N^{3} / f(N)\right)$ such that if

$$
\sum_{n \geq 1} \frac{1}{n f(n)}
$$

diverges, then for Lebesgue almost all $\alpha \in[0,1]$

$$
\limsup _{N \rightarrow \infty} R([-s, s], \alpha, N)=\infty
$$

\footnotetext{
2 This problem was posed at the problem session of the ELAZ conference in 2016.
} 
holds for any $s>0$; additionally, if (4) converges and $\sup \left\{f(2 x) / f(x): x \geq x_{0}\right\}$ is strictly less than 2 for some $x_{0}>0$, then NPPC $\left(\left(a_{n}\right)_{n}\right)$ has Hausdorff dimension at least $(1+\lambda(f))^{-1}$ where

$$
\lambda(f):=\liminf _{x \rightarrow \infty} \frac{\log f(x)}{\log x}
$$

denotes the lower order of infinity of $f$.

We record an immediate consequence of Theorem 2 by using the convention that the $r$-folded iterated logarithm is denoted by $\log _{r}(x)$, i.e.

$$
\log _{r}(x):=\log _{r-1}(\log (x))
$$

and $\log _{1}(x):=\log (x)$.

Corollary 1 Let $r$ be a positive integer. Then, there is a strictly increasing sequence $\left(a_{n}\right)_{n}$ of positive integers with

$$
E\left(A_{N}\right)=\Theta\left(\frac{N^{3}}{\log (N) \log _{2}(N) \ldots \log _{r}(N)}\right)
$$

such that $\operatorname{NPPC}\left(\left(a_{n}\right)_{n}\right)$ has full Lebesgue measure. Moreover, for any $\varepsilon>0$ there is a strictly increasing sequence $\left(a_{n}\right)_{n}$ of positive integers with

$$
E\left(A_{N}\right)=\Theta\left(\frac{\left(\log _{r}(N)\right)^{-\varepsilon} N^{3}}{\log (N) \log _{2}(N) \ldots \log _{r}(N)}\right)
$$

such that $\operatorname{NPPC}\left(\left(a_{n}\right)_{n}\right)$ has full Hausdorff dimension.

The proof of Theorem 2 connects the metric PPC property to the notion of optimal regular systems from Diophantine approximation. It uses, among other things, a Khintchine-type theorem due to Beresnevich. Furthermore, despite leading to better bounds, the nature of the sequences underpinning Theorem 2 is much simpler than the nature of those sequences previously constructed by Bourgain [2] (who used, inter alia, large deviations inequalities from probability theory), or the sequence of prime numbers studied by Walker [22] (who relied on estimates, derived by the circle-method, on the exceptional set in Goldbach-like problems).

\section{First main theorem}

Let us give an outline of the proof of Theorem 1. For doing so, we begin by sketching the reasoning of Theorem B: As it turns out, except for a set of negligible measure, the counting function in (1) can be written as a function (of $\alpha$ ) that admits a non-trivial estimate for its $L^{1}$-mean value. The mean value is infinitely often too small on sets whose measure is uniformly bounded from below. Thus, there exists a sequence of 
sets $\left(\Omega_{r}\right)_{r}$ of $\alpha \in[0,1]$ such that $R([-s, s], \alpha, N)$ is too small for every $\alpha \in \Omega_{r}$ for having PPC and Theorem B follows.

Our reasoning for proving Theorem 1 is building upon this argument of Bourgain while we introduce new ideas to construct a sequence of sets $\left(\Omega_{r}\right)_{r}$ that are "pairwise quasi independent"- meaning that for every fixed $t$ the relation

$$
\lambda\left(\Omega_{r} \cap \Omega_{t}\right) \leq \lambda\left(\Omega_{r}\right) \lambda\left(\Omega_{t}\right)+o(1)
$$

holds as $r \rightarrow \infty$ where $\lambda$ is the Lebesgue measure. Roughly speaking, applying a suitable version of the Borel-Cantelli lemma, combined with a sufficiently careful treatment of the $o$ (1) term, will then yield Theorem 1. However, before proceeding with the details of the proof we collect in the next paragraph some tools from additive combinatorics that are needed.

\subsection{Preliminaries}

We start with a well-know result relating, in a quantitative manner, the additive energy of a set of integers with the existence of a (relatively) dense subset with small difference set where the difference set $B-B:=\left\{b-b^{\prime}: b, b^{\prime} \in B\right\}$ for a set $B \subseteq \mathbb{R}$.

Lemma 1 (Balog-Szeméredi-Gowers lemma, [20, Thm 2.29]) Let $A \subseteq \mathbb{Z}$ be a finite set of integers. For any $c>0$ there exist $c_{1}, c_{2}>0$ depending only on $c$ such that the following holds. If $E(A) \geq c(\# A)^{3}$, then there is a subset $B \subseteq A$ such that

1. $\# B \geq c_{1} \# A$,

2. $\#(B-B) \leq c_{2} \# A$.

Moreover, we recall that for $\delta>0$ and $d \in \mathbb{Z}$ the set

$$
B(d, \delta):=\{\alpha \in[0,1]:\|d \alpha\| \leq \delta\}
$$

is called Bohr set. The following two simple observations will be useful.

Lemma 2 Let $B \subseteq \mathbb{Z}$ be a finite set of integers. Then,

$$
\lambda\left(\left\{\alpha \in[0,1]: \min _{d \in(B-B) \backslash\{0\}}\|d \alpha\|<\frac{\varepsilon}{\#(B-B)}\right\}\right) \leq 2 \varepsilon
$$

for every $\varepsilon \in(0,1)$.

Proof By observing that the set under consideration is contained in

$$
\bigcup_{\substack{m, n \in B \\ m \neq n}} B\left(m-n, \frac{\varepsilon}{\#(B-B)}\right)
$$


and

$$
\lambda\left(B\left(m-n, \frac{\varepsilon}{\#(B-B)}\right)\right)=\frac{2 \varepsilon}{\#(B-B)},
$$

the claim follows at once.

Lemma 3 Suppose A is a finite intersection of Bohr sets, and B is a finite union of Bohr sets. Then, $A \backslash B$ is the union of finitely many intervals.

Furthermore, we shall use the Borel-Cantelli lemma in a version due to Erdős, and Rényi.

Lemma 4 (Erdős-Rényi, cf. [8, Lem. 2.3]). Let $\left(A_{n}\right)_{n}$ be a sequence of Lebesgue measurable sets in $[0,1]$ satisfying

$$
\sum_{n \geq 1} \lambda\left(A_{n}\right)=\infty
$$

Then,

$$
\lambda\left(\limsup _{n \rightarrow \infty} A_{n}\right) \geq \limsup _{N \rightarrow \infty} \frac{\left(\sum_{n \leq N} \lambda\left(A_{n}\right)\right)^{2}}{\sum_{m, n \leq N} \lambda\left(A_{n} \cap A_{m}\right)} .
$$

Moreover, let us explain the main steps in the proof of Theorem 1. Let

$$
\varepsilon:=\varepsilon(j):=\frac{1}{10^{j}} c_{1}^{2}
$$

where $c_{1}>0$ is a constant to be specified later-on, and $j$ denotes a positive integer. In the first part of the argument, we show how a sequence-that is constructed in the second part of the argument—can be used to deduce Theorem 1. For every fixed $j$, we find a corresponding $s=s(j)$ and construct inductively a sequence $\left(\Omega_{r}\right)_{r}$ of exceptional values $\alpha$ with the following properties:

(i) For all $\alpha \in \Omega_{r}$, the pair correlation function admits the upper bound

$$
R([-s, s], \alpha, N) \leq 2 \tilde{c} s
$$

for some absolute constant $\tilde{c} \in(0,1)$, depending on $\left(a_{n}\right)_{n}$ only.

(ii) For all integers $r>t \geq 1$, the relation

$$
\lambda\left(\Omega_{r} \cap \Omega_{t}\right) \leq \lambda\left(\Omega_{r}\right) \lambda\left(\Omega_{t}\right)+2 \varepsilon \lambda\left(\Omega_{t}\right)+\mathcal{O}\left(r^{-2}\right)
$$

holds.

(iii) Each $\Omega_{r}$ is the union of finitely many intervals (hence measurable). 
(iv) For all $r \geq 1$, the measure $\lambda\left(\Omega_{r}\right)$ is uniformly bounded from below by

$$
\lambda\left(\Omega_{r}\right) \geq \frac{c_{1}^{2}}{8} .
$$

\subsection{Proof of Theorem 1}

1. Suppose there is $\left(\Omega_{r}\right)_{r}$ satisfying (i)-(iv). Then, by using (7), we get

$$
\begin{aligned}
\sum_{r, t \leq N} \lambda\left(\Omega_{r} \cap \Omega_{t}\right) & \leq 2 \sum_{2 \leq t \leq N} \sum_{1 \leq r<t}\left(\lambda\left(\Omega_{r}\right) \lambda\left(\Omega_{t}\right)\right)+2 \varepsilon N^{2}+\mathcal{O}(N) \\
& \leq\left(\sum_{t \leq N} \lambda\left(\Omega_{t}\right)\right)^{2}+2 \varepsilon N^{2}+\mathcal{O}(N)
\end{aligned}
$$

By recalling that $\Omega_{r}=\Omega_{r}(\varepsilon)=\Omega_{r}(j)$, we let

$$
\Omega(j):=\limsup _{r \rightarrow \infty} \Omega_{r} .
$$

By using the inequality above in combination with Lemma 4 and the bound (8), we obtain that the set $\Omega(j)$ has measure at least

$$
\begin{aligned}
\limsup _{N \rightarrow \infty} \frac{\left(\sum_{r \leq N} \lambda\left(\Omega_{r}\right)\right)^{2}}{\sum_{r, t \leq N} \lambda\left(\Omega_{r} \cap \Omega_{t}\right)} & \geq \limsup _{N \rightarrow \infty} \frac{1}{1+\frac{4 \varepsilon N^{2}}{\left(\sum_{r \leq N} \lambda\left(\Omega_{r}\right)\right)^{2}}} \\
& \geq \limsup _{N \rightarrow \infty} \frac{1}{1+\frac{256}{c_{1}^{4}} \varepsilon}=\frac{1}{1+\frac{256}{c_{1}^{4}} \varepsilon} .
\end{aligned}
$$

Note that due to (6), for every $\alpha \in \Omega(j)$ the sequence $\left(\alpha a_{n}\right)_{n}$ does not have PPC. Now, letting $j \rightarrow \infty$ proves the assertion.

2. For constructing $\left(\Omega_{r}\right)_{r}$ with the required properties, let $c>0$ such that $E\left(A_{N}\right)>$ $c N^{3}$ for infinitely many integers $N$. By choosing an appropriate subsequence $\left(N_{i}\right)_{i}$ and omitting the subscript $i$ for ease of notation, we may suppose that $E\left(A_{N}\right)>c N^{3}$ holds for every $N$ occurring in this proof. Moreover, let $c_{1}, c_{2}$ and $B_{N}$ be as in Lemma 1 , corresponding to the $c$ just mentioned. Let

$$
s=\frac{\varepsilon}{2 c_{2}} .
$$

Arguing inductively, while postponing the base step, ${ }^{3}$ we assume that there are sets $\left(\Omega_{r}\right)_{1 \leq r<R}$ given that satisfy the properties (i)-(iv) for all distinct integers $1 \leq r, t<$ $R$. Let $N \geq R$. Since, due to Lemma 1 ,

\footnotetext{
3 The base step uses simplified versions of the arguments exploited in the induction step, and will therefore be postponed.
} 


$$
\frac{s}{N} \leq \frac{\varepsilon}{\#(B-B)}
$$

Lemma 2 implies that the set $\Omega_{\varepsilon, N}$ of all $\alpha \in[0,1]$ satisfying $\|(r-t) \alpha\|<N^{-1} s$ for some distinct $r, t \in B_{N}$ has measure at most $2 \varepsilon$. Setting

$$
\mathcal{D}_{N}:=\left\{(r, t) \in\left(A_{N} \times A_{N}\right) \backslash\left(B_{N} \times B_{N}\right): r \neq t\right\},
$$

we get for $\alpha \notin \Omega_{\varepsilon, N}$ that

$$
R([-s, s], \alpha, N)=\frac{1}{N} \#\left\{(r, t) \in \mathcal{D}_{N}:\|(r-t) \alpha\|<N^{-1} s\right\} .
$$

Let $\ell_{R}$ denote the length of the smallest subinterval of $\Omega_{r}$ for $1 \leq r<R$, and define $C\left(\Omega_{r}\right)$ to be the set of subintervals of $\Omega_{r}$. Note that $\ell_{R}>0$, and $\max _{1 \leq r<R} \# C\left(\Omega_{r}\right)<$ $\infty$. We divide $[0,1)$ into

$$
P:=\left\lfloor 1+2 \ell_{R}^{-1} R^{2} \max _{1 \leq r<R} \# C\left(\Omega_{r}\right)\right\rfloor
$$

parts $\mathcal{P}_{i}$ of equal lengths (where $\lfloor x\rfloor$ is the integer part of $x$ ), i.e.

$$
\mathcal{P}_{i}:=\left[\frac{i}{P}, \frac{i+1}{P}\right)
$$

where $i=0, \ldots, P-1$. Let $\mathbf{1}_{X}$ denote the characteristic function of a Borel set $X \subseteq[0,1]$. After writing

$$
\begin{array}{r}
\frac{1}{N} \int_{\mathcal{P}_{i}} \#\left\{(r, t) \in \mathcal{D}_{N}:\|(r-t) \alpha\| \leq N^{-1} s\right\} \mathrm{d} \alpha \\
=\frac{1}{N} \sum_{(r, t) \in \mathcal{D}_{N}} \int_{\mathcal{P}_{i}} \mathbf{1}_{\left[-\frac{s}{N}, \frac{s}{N}\right]}(\|(r-t) \alpha\|) \mathrm{d} \alpha,
\end{array}
$$

we split the sum into two parts: one part containing differences $|r-t|>R^{k} P$, and a second part containing differences $|r-t| \leq R^{k} P$ where

$$
k:=\left\lfloor\frac{1}{\log 2} \log \frac{8(4 s+1)}{\left(c_{1}^{2}-2^{-1} c_{1}^{4}\right) s}\right\rfloor+1 .
$$

The Cauchy-Schwarz inequality implies

$$
\int_{\mathcal{P}_{i}} \mathbf{1}_{\left[0, \frac{s}{N}\right]}(\|(r-t) \alpha\|) \mathrm{d} \alpha \leq \sqrt{\frac{1}{P} \frac{2 s}{N}} .
$$


Since for any $x>0$ there are at most $2 x N$ choices of $(r, t) \in \mathcal{D}_{N}$ such that $|r-t| \leq x$, we obtain

$$
\frac{1}{N} \sum_{\substack{(r, t) \in \mathcal{D}_{N} \\|r-t| \leq P R^{k}}} \int_{\mathcal{P}_{i}} \mathbf{1}_{\left[0, \frac{s}{N}\right]}(\|(r-t) \alpha\|) \mathrm{d} \alpha \leq 2 P R^{k} \sqrt{\frac{1}{P} \frac{2 s}{N}}
$$

which is $\leq P^{-1} R^{-k}$ if $N$ is sufficiently large. Moreover, for any $|r-t|>P R^{k}$ we observe that

$$
\begin{aligned}
& \int_{\mathcal{P}_{i}} \mathbf{1}_{\left[0, \frac{s}{N}\right]}(\|(r-t) \alpha\|) \mathrm{d} \alpha \\
& \leq \frac{2 s}{N|r-t|}\left(\#\left\{j \leq|r-t|: j /|r-t| \in \mathcal{P}_{i}\right\}+1\right) \leq \frac{2 s}{P N}+\frac{4 s}{P R^{k} N}
\end{aligned}
$$

Also note that $\# \mathcal{D}_{N} \leq N^{2}-\left(\# B_{N}\right)^{2} \leq \tilde{c} N^{2}$ where $\tilde{c}:=1-c_{1}^{2}$. Therefore, the mean value (9) of the modified pair correlation counting function on the interval $\mathcal{P}_{i}$ admits the upper bound

$$
\frac{1}{N}\left(\# \mathcal{D}_{N}\right)\left(\frac{2 s}{P N}+\frac{4 s}{P R^{k} N}\right)+\frac{1}{P R^{k}} \leq \frac{2 \tilde{c} s}{P}+\frac{4 s+1}{P R^{k}}
$$

Hence, it follows that the measure of the set $\Delta_{N}(i)$ of $\alpha \in \mathcal{P}_{i}$ with

$$
\frac{1}{N} \#\left\{(r, t) \in \mathcal{D}_{N}:\|(r-t) \alpha\| \leq N^{-1} s\right\} \leq 2\left(1-\frac{c_{1}^{2}}{2}\right) s
$$

admits, by the choice of $k$, the lower bound

$$
\lambda\left(\Delta_{N}(i)\right) \geq \frac{1}{P}-\frac{1}{P} \frac{2 \tilde{c} s+(4 s+1) R^{-k}}{2\left(1-\frac{c_{1}^{2}}{2}\right) s} \geq \frac{1}{P}\left(\frac{c_{1}^{2}}{2}-\frac{c_{1}^{2}}{8}\right) .
$$

Note that $\Delta_{N}(i)$ is the union of finitely many intervals, due to Lemma 3. So, we may take $\Delta_{N}^{\prime}(i) \subset \Delta_{N}(i)$ being a finite union of intervals such that $\lambda\left(\Delta_{N}^{\prime}(i)\right)$ equals the lower bound in (11). Let

$$
\Omega_{R}:=\Omega_{R}(N):=\Delta_{N} \backslash \Omega_{\varepsilon, N} \text { where } \Delta_{N}:=\bigcup_{i=0}^{P-1} \Delta_{N}^{\prime}(i)
$$


We are going to show now that $\Omega_{R}$ satisfies the properties (i)-(iv). Now, $\Omega_{R}$ satisfies property (iv) with $r=R$ since

$$
\lambda\left(\Omega_{R}\right) \geq \lambda\left(\Delta_{N}\right)-\lambda\left(\Omega_{\varepsilon, N}\right)=\frac{c_{1}^{2}}{2}-\frac{c_{1}^{2}}{8}-2 \varepsilon \geq \frac{c_{1}^{2}}{8} .
$$

Furthermore, $\Omega_{R}$ satisfies property (i) by construction and also property (iii) since all sets involved in the construction of $\Omega_{R}$ were a finite union of intervals. Let $1 \leq r<R$, and $I$ be a subinterval of $\Omega_{r}$. Then,

$$
\begin{aligned}
\lambda\left(I \cap \Delta_{N}\right)= & \sum_{i: \mathcal{P}_{i} \cap I \neq \emptyset} \lambda\left(\mathcal{P}_{i} \cap I \cap \Delta_{N}\right) \leq \frac{2}{P}+\sum_{i: \mathcal{P}_{i} \subsetneq I} \lambda\left(\mathcal{P}_{i} \cap \Delta_{N}\right) \leq \frac{2}{P} \\
& +\sum_{i: \mathcal{P}_{i} \subsetneq I} \lambda\left(\Delta_{N}^{\prime}(i)\right) .
\end{aligned}
$$

By summing over all subintervals $I \in C\left(\Omega_{r}\right)$, we obtain that

$$
\begin{gathered}
\lambda\left(\Omega_{r} \cap \Delta_{N}\right) \leq \sum_{I \in C\left(\Omega_{r}\right)}\left(\frac{2}{P}+\sum_{i: \mathcal{P}_{i} \subsetneq I} \lambda\left(\Delta_{N}^{\prime}(i)\right)\right) \leq \frac{1}{R^{2}} \\
+\sum_{I \in C\left(\Omega_{r}\right)} P \lambda(I) \frac{\lambda\left(\Delta_{N}\right)}{P}=\lambda\left(\Omega_{r}\right) \lambda\left(\Delta_{N}\right)+\frac{1}{R^{2}} .
\end{gathered}
$$

We deduce property (ii) from this estimate and Lemma 2 via

$$
\begin{aligned}
\lambda\left(\Omega_{r} \cap \Omega_{R}\right) & \leq \lambda\left(\Omega_{r} \cap \Delta_{N}\right) \\
& \leq \lambda\left(\Omega_{r}\right)\left(\lambda\left(\Delta_{N}\right)-\lambda\left(\Omega_{\varepsilon, N}\right)\right)+R^{-2}+\lambda\left(\Omega_{r}\right) \lambda\left(\Omega_{\varepsilon, N}\right) \\
& \leq \lambda\left(\Omega_{r}\right) \lambda\left(\Omega_{R}\right)+2 \varepsilon \lambda\left(\Omega_{r}\right)+R^{-2} .
\end{aligned}
$$

This concludes the induction step. The only part missing now is the base step of the induction. For realizing it, let $N$ denote the smallest integer $m$ with $E\left(A_{m}\right)>\mathrm{cm}^{3}$. We replace $\mathcal{P}_{i}$ in $(9)$ by $[0,1]$ to directly derive

$$
\int_{0}^{1} \frac{1}{N} \#\left\{(r, t) \in \mathcal{D}_{N}:\|(r-t) \alpha\| \leq N^{-1} s\right\} \mathrm{d} \alpha \leq 2 \tilde{c} s,
$$

and conclude that the set $\Omega_{1}^{\prime}$ of $\alpha \in[0,1]$ satisfying (10) has a measure at least $c_{1}^{2} / 2$. Thus, $\Omega_{1}:=\Omega_{1}^{\prime} \backslash \Omega_{N, \varepsilon}$ has measure at least as large as the right hand side of (8). For property (7), there is nothing to check and that $\Omega_{1}$ is a finite union of intervals follows from Lemma 3 by observing that

$$
\Omega_{1}^{\prime}=\bigcap_{d_{1}, \ldots, d_{\lfloor N 2 \tilde{c} s\rfloor}}\left(B\left(d_{1}, N^{-1} s\right)^{C} \cup \cdots \cup B\left(d_{\lfloor N 2 \tilde{c} s\rfloor}, N^{-1} s\right)^{C}\right)
$$


where the intersection runs through any set of $\lfloor N 2 \tilde{c} s\rfloor$-tuples of differences $d_{i}=$ $r_{i}-t_{i} \neq 0$ of components of $\left(r_{i}, t_{i}\right) \in \mathcal{D}_{N}$ for $i=1, \ldots,\lfloor N 2 \tilde{c} s\rfloor$.

Thus, the proof is complete.

\section{Second main theorem}

The sequences $\left(a_{n}\right)_{n}$ enunciated in Theorem 2 are constructed in two steps. In the first step, we concatenate (finite) blocks, with suitable lengths, of arithmetic progressions to form a set $P_{A}$. In the second step, we concatenate (finite) blocks, with suitable lengths, of geometric progressions to form a set $P_{G}$ and then define $a_{n}$ to be the $n$-th smallest element of $P_{A} \cup P_{G}$. On the one hand, the arithmetic progression like part $P_{A}$ serves to ensure, due to considerations from metric Diophantine approximation, the divergence property (5) on a set with full measure or controllable Hausdorff dimension; on the other hand, the geometric progression like part $P_{G}$ lowers the additive energy, as much as it can. For doing so, a geometric block will appear exactly before and after an arithmetic block, and have much more elements.

For writing the construction precisely down, we introduce some notation. Suppose throughout this section that $f$ is as in Theorem 2 . We set $P_{A}^{(1)}$ to be the empty set while $P_{G}^{(1)}:=\{1,2\}$. Suppose $P_{A}^{(j-1)}, P_{G}^{(j-1)}$ for $j \geq 2$ are already constructed. Let $C_{j}=2 \max \left\{P_{G}^{(j-1)}\right\}$. Then

$$
P_{A}^{(j)}:=\left\{C_{j}+h: 1 \leq h \leq\left\lfloor\left(f\left(2^{j}\right)\right)^{-\beta} 2^{j}\right\rfloor\right\}
$$

and $P_{G}^{(j)}$ is defined via

$$
P_{G}^{(j)}:=\left\{2 C_{j}+2^{i}: 1 \leq i \leq\left\lfloor\left(f\left(2^{j}\right)\right)^{-\gamma} 2^{j}\left(1-\left(f\left(2^{j}\right)\right)^{\gamma-\beta}\right)\right\rfloor\right\}
$$

where $0<\gamma<\beta<3 / 4$ are parameters ${ }^{4}$ to be chosen later-on. Letting

$$
P_{A}:=\bigcup_{j \geq 1} P_{A}^{(j)}, \quad P_{G}:=\bigcup_{j \geq 1} P_{G}^{(j)},
$$

we denote by $a_{n}$ the $n$-th smallest element in $P_{A} \cup P_{G}$. For $d \in \mathbb{Z}$ and finite sets of integers $X, Y$, we abbreviate the number of representations of $d$ as a difference of an $x \in X$ and a $y \in Y$ by

$$
\operatorname{rep}_{X, Y}(d):=\#\{(x, y) \in X \times Y: x-y=d\}
$$

\footnotetext{
${ }^{4}$ No particular importance should be attached to requiring $\beta<3 / 4$, or using "dyadic steps lengths $2^{j}$ ". Doing so is for simplifying the technical details only —eventually, it will turn out that $\beta=2 / 3=2 \gamma$ is the optimal choice of parameters in this approach. For proving this to the reader, we leave $\gamma, \beta$ undetermined till the end of this section.
} 
for later reference, we record here that the additive energy of a set $X$ and the pair correlation counting function can be written as

$$
E(X)=\sum_{d \in \mathbb{Z}}\left(\operatorname{rep}_{X, X}(d)\right)^{2}
$$

and

$$
R([-s, s], \alpha, N)=\frac{1}{N} \sum_{d \in \mathbb{Z} \backslash\{0\}} \operatorname{rep}_{A_{N}, A_{N}}(d) \mathbf{1}_{\left[0, \frac{s}{N}\right]}(\|\alpha d\|)
$$

\subsection{Preliminaries}

For determining the order of magnitude of $E\left(A_{N}\right)$, the following considerations are useful. Since the cardinality $P_{G}^{(j)} \cup P_{A}^{(j)}$ has about exponential growth, it is reasonable to expect $E\left(A_{N}\right)$ to be of the same order of magnitude as the additive energy of the last block $P_{G}^{(J)} \cup P_{A}^{(J)}$ that is fully contained in $A_{N}$-note that $J=J(N)$; i.e. to expect the magnitude of $E\left(P_{G}^{(J)} \cup P_{A}^{(J)}\right)$ which is roughly $E\left(P_{A}^{(J)}\right)$. The next proposition verifies this heuristic.

Proposition 1 Let $\left(a_{n}\right)_{n}$ be as in the beginning of Sect. 3, and $f$ be as in one of the two assertions in Theorem 2. Then, $E\left(A_{N}\right)=\Theta\left(N^{3}(f(N))^{-3(\beta-\gamma)}\right)$.

For the proof of Proposition 1, we need the following technical lemma.

Lemma 5 Let $F_{j}:=2^{j}\left(f\left(2^{j}\right)\right)^{-\delta}$, for $j \geq 1$ and fixed $\delta \in(0,1)$, where $f$ is as in Proposition 1. Then, $\sum_{i \leq j} F_{i}=\mathcal{O}\left(F_{j}\right)$ and

$$
\sum_{d \in \mathbb{Z}}\left(\sum_{j, i \leq J} \operatorname{rep}_{P_{G}^{(j)}, P_{A}^{(i)}}(d)\right)^{2}=\mathcal{O}\left(J^{6} 2^{2 J}\right) .
$$

Proof Suppose that $f(x)=\mathcal{O}\left(x^{1 / 3}(\log x)^{-7 / 3}\right)$ is such that (4) diverges. Because

$$
\sum_{j \leq J+1} \frac{1}{f\left(2^{j}\right)} \geq \sum_{k \leq 2^{J}} \frac{1}{k f(k)}
$$

diverges as $J \rightarrow \infty$ and $\left(f\left(2^{j}\right) / f\left(2^{j+1}\right)\right)_{j}$ is non-decreasing, we conclude that $\lim _{j \rightarrow \infty}\left(f\left(2^{j}\right) / f\left(2^{j+1}\right)\right)=1$. Therefore, there is an $i_{0}$ such that the estimate

$$
\left(f\left(2^{i}\right)\right)^{-1} f\left(2^{i+h}\right)<(3 / 2)^{\frac{h}{\delta}}
$$

holds for any $i \geq i_{0}$ and $h \in \mathbb{N}$. Hence,

$$
\frac{1}{F_{j}} \sum_{i \leq j} F_{i} \leq o(1)+\sum_{i_{0} \leq i \leq j} 2^{i-j}\left(\frac{3}{2}\right)^{j-i}=\mathcal{O}(1) .
$$


If $f$ is such that (4) converges and $f(2 x) \leq(2-\varepsilon) f(x)$ for $x$ large enough, then we obtain by a similar argument that $\sum_{i \leq j} F_{i}$ is in $\mathcal{O}\left(F_{j}\right)$. Furthermore, rep $P_{G}^{(j)}, P_{A}^{(i)}(d)=$ $\mathcal{O}(i)$, for every $j \geq 1$, and non-vanishing for $\mathcal{O}\left(2^{2 j}\right)$ values of $d$ which implies the last claim.

We can now prove the proposition.

Proof of Proposition 1 Let $N \geq 1$ be large and denote by $J=J(N) \geq 0$ the greatest integer $j$ such that $P_{G}^{(j-1)} \subseteq A_{N}$. By exploiting (12),

$$
E\left(A_{N}\right) \geq E\left(P_{A}^{(J-1)}\right) \gg\left(\# P_{A}^{(J-1)}\right)^{3}
$$

which is seen to be $\gg f(N)^{-3(\beta-\gamma)} N^{3}$. Hence, it remains to show that $E\left(A_{N}\right)=$ $\mathcal{O}\left((f(N))^{-3(\beta-\gamma)} N^{3}\right)$. Note that

$$
E\left(A_{N}\right) \leq \sum_{d \in \mathbb{Z}}\left(\operatorname{rep}_{A_{T_{J}}, A_{T_{J}}}(d)\right)^{2} \quad \text { where } T_{J}:=\# \bigcup_{j \leq J}\left(P_{A}^{(j)} \cup P_{G}^{(j)}\right)
$$

Moreover, $\operatorname{rep}_{A_{T_{J}}, A_{T_{J}}}(d)=S_{1}(d)+S_{2}(d)$ where $S_{2}(d)$ denotes the mixed sum $\sum_{i, j \leq J}\left(\operatorname{rep}_{P_{A}^{(j)}, P_{G}^{(i)}}(d)+\operatorname{rep}_{P_{G}^{(i)}, P_{A}^{(j)}}(d)\right)$, and $S_{1}(d)$ abbreviates $\sum_{i, j \leq J}\left(\operatorname{rep}_{P_{G}^{(i)}, P_{G}^{(j)}}\right.$ $\left.(d)+\operatorname{rep}_{P_{A}^{(i)}, P_{A}^{(j)}}(d)\right)$. Using that for any $a, b \in \mathbb{R}$ the inequality $(a+b)^{2} \leq 2\left(a^{2}+b^{2}\right)$ holds, we obtain

$$
E\left(A_{N}\right)=\mathcal{O}\left(\sum_{d \in \mathbb{Z}}\left(S_{1}(d)\right)^{2}+\sum_{d \in \mathbb{Z}}\left(S_{2}(d)\right)^{2}\right) .
$$

Lemma 5 implies that $\sum_{d \in \mathbb{Z}}\left(S_{2}(d)\right)^{2}=\mathcal{O}\left((\log N)^{6} N^{2}\right)$ due to $J=\mathcal{O}(\log N)$. Furthermore letting $F_{j}=2^{j}\left(f\left(2^{j}\right)\right)^{-\beta}$, we observe that $\operatorname{rep}_{P_{A}^{(i)}, P_{A}^{(j)}}(d)$ is non-vanishing for at most $4 F_{J}$ values of $d$ as $i, j \leq J$. Since $\operatorname{rep}_{P_{A}^{(i)}, P_{A}^{(j)}}(d) \leq F_{\min (i, j)}$ holds, we deduce that

$$
\sum_{i, j \leq J} \operatorname{rep}_{P_{A}^{(i)}, P_{A}^{(j)}}(d)=\mathcal{O}\left(\sum_{j \leq J} \sum_{i \leq j} F_{i}\right)=\mathcal{O}\left(F_{J}\right)
$$

Since $\operatorname{rep}_{P_{G}^{(i)}, P_{G}^{(j)}}(d) \leq 1$, as $i, j \leq J$, is non-zero for at most $\mathcal{O}\left(T_{J}^{2}\right)=\mathcal{O}\left(N^{2}\right)$ values of $d$, we obtain that

$$
\sum_{d \in \mathbb{Z}}\left(S_{1}(d)\right)^{2}=\mathcal{O}\left(F_{J}^{3}+(\log N)^{6} N^{2}\right)=\mathcal{O}\left(N^{3}(f(N))^{-3(\beta-\gamma)}\right)
$$

Hence, $E\left(A_{N}\right)=\mathcal{O}\left(N^{3}(f(N))^{-3(\beta-\gamma)}\right)$. 
For estimating the measure or the Hausdorff dimension of NPPC $\left(\left(a_{n}\right)_{n}\right)$ from below, we recall the notion of an optimal regular system. This notion, roughly speaking, describes sequences of real numbers that are exceptionally well distributed in any subinterval, in a uniform sense, of a fixed interval.

Definition Let $J$ be a bounded real interval, and $S=\left(\alpha_{i}\right)_{i}$ a sequence of distinct real numbers. $S$ is called an optimal regular system in $J$ if there exist constants $c_{1}, c_{2}, c_{3}>$ 0 - depending on $S$ and $J$ only-such that for any interval $I \subseteq J$ there is an index $Q_{0}=Q_{0}(S, I)$ such that for any $Q \geq Q_{0}$ there are indices

$$
c_{1} Q \leq i_{1}<i_{2}<\cdots<i_{t} \leq Q
$$

satisfying $\alpha_{i_{h}} \in I$ for $h=1, \ldots, t$, and

$$
\left|\alpha_{i_{h}}-\alpha_{i_{\ell}}\right| \geq \frac{c_{2}}{Q}
$$

for $1 \leq h \neq \ell \leq t$, and

$$
c_{3} \lambda(I) Q \leq t \leq \lambda(I) Q .
$$

Moreover, we need the following result(s) due to Beresnevich which may be thought of as a far reaching generalization of the classical Khintchine theorem, and the JarníkBesicovitch theorem in Diophantine approximation.

Theorem 3 [7, Thm. 6.1, Thm. 6.2] Suppose $\psi: \mathbb{R}_{>0} \rightarrow \mathbb{R}_{>0}$ is a continuous, nonincreasing function, and $S=\left(\alpha_{i}\right)_{i}$ an optimal regular system in $(0,1)$. Let $\mathcal{K}_{S}(\psi)$ denote the set of $\xi$ in $(0,1)$ such that $\left|\xi-\alpha_{i}\right|<\psi(i)$ holds for infinitely many $i$. If

$$
\sum_{n \geq 1} \psi(n)
$$

diverges, then $\mathcal{K}_{S}(\psi)$ has full measure.

Conversely, if (17) converges, then $\mathcal{K}_{S}(\psi)$ has measure zero and the Hausdorff dimension equals the reciprocal of the lower order of $\frac{1}{\psi}$ at infinity.

For a rational $\alpha=\frac{p}{q}$, where $p, q \in \mathbb{Z}, q \neq 0$, we denote by $H(\alpha)$ its (naive) height, i.e. $H(\alpha):=\max \{|p|,|q|\}$. It is well-known that the set of rational numbers in $(0,1)$ - first running through all rationals of height 1 ordered by increasing numerical value, then through all rationals with height 2 ordered by increasing numerical value, and so on-gives rise to an optimal regular system in $(0,1)$. The following lemma says, roughly speaking, that this assertion remains true for the set of rationals in $(0,1)$ whose denominators are members of a special sequence that is not too sparse in the natural numbers, and hand-tailored for our purposes. The proof can be given by modifying the proof of the classical case, compare [7, Prop. 5.3]; however, we shall give the details for making this article more self-contained. 
Lemma 6 Let $\vartheta: \mathbb{R}_{>0} \rightarrow \mathbb{R}_{>1}$ be monotonically increasing to infinity with $\vartheta(x)=$ $\mathcal{O}\left(x^{1 / 4}\right)$ and $\vartheta\left(2^{j+1}\right) / \vartheta\left(2^{j}\right) \rightarrow 1$ as $j \rightarrow \infty$. For each $j \in \mathbb{N}$, we let

$$
B_{j}:=\frac{2^{j}}{f\left(2^{j}\right) \sqrt{\vartheta\left(2^{j}\right)}}, \quad b_{j}:=\frac{2}{3} B_{j}
$$

Let $S=\left(\alpha_{i}\right)_{i}$ denote a sequence running through all rationals in $(0,1)$ whose denominators are in $M:=\bigcup_{j \geq 1}\left\{n \in \mathbb{N}: b_{j} \leq n \leq B_{j}\right\}$ such that $i \mapsto H\left(\alpha_{i}\right)$ is non-decreasing. Then, $S$ is an optimal regular system in $(0,1)$.

Proof Let $X \geq 2$. There are strictly less than $2 X^{2}$ rational numbers in $(0,1)$ with height bounded by $X$. We take $J=J(X)$ to be the largest integer $j \geq 1$ such that $B_{j} \leq X$. Then, for $X$ large enough, there are at least, due to a basic property of the Eulerian totient function,

$$
\begin{gathered}
\sum_{j \leq J} \sum_{b_{j} \leq q \leq B_{j}} \varphi(q) \geq \sum_{j \leq J}\left(\frac{1}{3 \pi^{2}} B_{j}^{2}+\mathcal{O}\left(B_{j} \log B_{j}\right)\right) \\
\geq \frac{1}{6 \pi^{2}} \frac{2^{2 J}}{f^{2}\left(2^{J}\right) \vartheta\left(2^{J}\right)}+\mathcal{O}\left(J 2^{J}\right)>\left(\frac{X}{5 \pi}\right)^{2}
\end{gathered}
$$

distinct such rationals in $(0,1)$ with height not exceeding $X$. Hence, we obtain

$$
\frac{\sqrt{i}}{2} \leq H\left(\alpha_{i}\right) \leq \sqrt{25 \pi^{2}(i+1)}+1
$$

for $i$ sufficiently large. Let $Q \in \mathbb{N}, I \subseteq[0,1]$ be a non-empty interval, and let $F$ denote the set of $\xi \in I$ satisfying the inequality $\|q \xi\|<Q^{-1}$ with some $1 \leq q \leq \frac{1}{1000} Q$. Note that $F$ has measure at most

$$
\sum_{q \leq \frac{1}{1000} Q}\left(\frac{2}{q Q} q \lambda(I)+\frac{2}{q Q}\right)=\frac{1}{500} \lambda(I)+\mathcal{O}\left(\frac{\log Q}{Q}\right)<\frac{1}{400} \lambda(I)
$$

for $Q \geq Q_{0}$ where $Q_{0}=Q_{0}(S, I)$ is sufficiently large. Let $\left\{p_{j} / q_{j}\right\}_{1 \leq j \leq t}$ be the set of all rationals $p_{j} / q_{j} \in(0,1)$ with $q_{j} \in M, \frac{1}{1000} Q<q_{j}<Q$ that satisfy

$$
\left|\frac{p_{j}}{q_{j}}-\frac{p_{j^{\prime}}}{q_{j^{\prime}}}\right|>\frac{2000}{Q^{2}}
$$

whenever $1 \leq j \neq j^{\prime} \leq t$. Observe that for $J$ as above with $X=Q$ sufficiently large, it follows that

$$
\left\{q \in M: b_{J} \leq q \leq B_{J}\right\} \subseteq\left\{\left\lfloor\frac{Q}{1000}\right\rfloor,\left\lfloor\frac{Q}{1000}\right\rfloor+1, \ldots, Q\right\}
$$


holds and hence, there are at least

$$
\frac{1}{3 \pi^{2}} B_{J}^{2}+\mathcal{O}\left(B_{J} \log B_{J}\right)>\frac{1}{400} Q^{2}
$$

choices of $p_{j} / q_{j} \in(0,1)$ with $q_{j} \in M$ and $\frac{1}{1000} Q<q_{j}<Q$. Due to $\lambda(I \backslash F)>$ $\frac{399}{400} \lambda(I)$, we conclude

$$
t \geq 400 \frac{Q^{2}}{4000} \frac{399}{400} \lambda(I) .
$$

Thus, taking $c_{1}:=1 / 1000, c_{2}:=2000$, and $c_{3}:=\frac{399}{4000}$ in (14)-(16), respectively, $S$ is shown to be an optimal regular system.

Now we can proceed to the proof of Theorem 2.

\subsection{Proof of Theorem 2}

We argue in two steps depending on whether or not the series (4) converges. Proposition (1) implies the announced $\Theta$-bounds on the additive energy of $A_{N}$, in both cases.

(i) Suppose (4) diverges, and fix $s>0$. Let $\vartheta: \mathbb{R}_{>0} \rightarrow \mathbb{R}_{>1}$ be monotonically increasing to infinity with $\vartheta(x)=\mathcal{O}\left(x^{1 / 4}\right)$ such that

$$
\psi(n):=\frac{1}{n f(n) \vartheta(n)}
$$

satisfies the divergence condition (17). Thus, $\vartheta\left(2^{j}\right) / \vartheta\left(2^{j-1}\right) \rightarrow 1$ as $j \rightarrow \infty$, and $S=\left(\alpha_{i}\right)_{i}$ from Lemma 6 is an optimal regular system. Furthermore, if $b_{J} \leq n \leq B_{J}$, for some integer $J$, then, by the properties of $\vartheta$ from Lemma 6 and the relation $\sum_{j \leq J} F_{j}=\mathcal{O}\left(F_{J}\right)$ from Lemma 5, we conclude that

$$
\sum_{j \leq J-1} \sum_{b_{j} \leq m \leq B_{j}} \varphi(m)=\Theta\left(B_{J}^{2}\right)
$$

implies that $\alpha_{i}=m / n$ entails $i \geq c n^{2}$ where $c=c(f, \vartheta)>0$ is a constant. Therefore, $\psi(i) \leq c^{-1} n^{-2}\left(f\left(c n^{2}\right) \vartheta\left(c n^{2}\right)\right)^{-1}$. The growth assumption on $f$ and $\vartheta(x)=\mathcal{O}\left(x^{1 / 4}\right)$ yields that if $j$ is large enough, then $b_{j} \leq n \leq B_{j}$ implies $c n^{2}>2^{j}$ and hence we obtain $\psi(i) \leq c^{-1} n^{-2}\left(f\left(2^{j}\right) \vartheta\left(2^{j}\right)\right)^{-1}$. Combining these considerations, we infer that

$$
n \psi(i)=\mathcal{O}\left(2^{-j}\left(\vartheta\left(2^{j}\right)\right)^{-1 / 2}\right)
$$


Applying Theorem 3 with $\psi$ as in (18), implies that $\mathcal{K}_{S}(\psi)$ has full Lebesgue measure. Therefore, for any $\alpha \in \mathcal{K}_{S}(\psi)$ we get

$$
\|n \alpha\| \leq n\left|\alpha-\alpha_{i}\right|=\mathcal{O}\left(2^{-j}\left(\vartheta\left(2^{j}\right)\right)^{-1 / 2}\right)
$$

for infinitely many $i$ and $j=j(i)$. Now if $b_{j} \leq n \leq B_{j}$ for $j$ sufficiently large and $n, \alpha$ as in (19), then it follows that by taking any integer $m \leq\left(f\left(2^{j}\right)\right)^{\gamma}\left(\vartheta\left(2^{j}\right)\right)^{1 / 3}$ that also the multiples

$$
n m \leq 2^{j}\left(f\left(2^{j}\right)\right)^{\gamma-1}\left(\vartheta\left(2^{j}\right)\right)^{-1 / 6}
$$

satisfy that $\mathbf{1}_{\left[0, s / T_{j}\right]}(\|\alpha(m n)\|)=1$ where $T_{j}=\mathcal{O}\left(2^{j}\left(f\left(2^{j}\right)\right)^{-\gamma}\right)$ is as in the proof of Proposition 1. If additionally $\gamma-1 \leq-\beta$ holds, then we obtain that

$$
\operatorname{rep}_{A_{T_{j}}, A_{T_{j}}}(m n) \geq 2^{j-1}\left(f\left(2^{j}\right)\right)^{-\beta}
$$

holds for $j$ sufficiently large. By (13), we obtain

$$
R\left([-s, s], \alpha, T_{j}\right) \geq C\left(f\left(2^{j}\right)\right)^{2 \gamma-\beta}\left(\vartheta\left(2^{j}\right)\right)^{1 / 3}
$$

for infinitely many $j$ where $C>0$ is some constant. For the optimal choice of the parameters $\beta, \gamma>0$, we are therefore led to maximize $\beta-\gamma$ where $2 \gamma-\beta \geq 0$ and $\gamma-1 \leq-\beta$ have to be satisfied. The solution is given if equality in the first inequality occurs, leading to $\beta=2 / 3$ and $\gamma=1 / 3$. Hence, (5) follows for $\alpha \in \mathcal{K}_{S}(\psi)$.

(ii) Suppose the series (4) converges. We keep the same sequence as in step (i) while taking $\vartheta(x)=1+\log (x)$, as we may. The arguments of step (i) show that any $\alpha \in \mathcal{K}_{S}(\psi)$ satisfies (5); now the conclusion is that $\mathcal{K}_{S}(\psi)$ has Hausdorff dimension at least equal to the reciprocal of

$$
\liminf _{x \rightarrow \infty} \frac{-\log (\psi(x))}{\log x}=1+\liminf _{x \rightarrow \infty} \frac{\log f(x)}{\log x} .
$$

Thus, the proof is complete.

\section{Concluding remarks}

It should be possible to relax the growth restriction $f(x)=\mathcal{O}\left(x^{1 / 3}(\log (x))^{-7 / 3}\right)$ in Theorem 2 on the expense of some additional technical work; as the main objective in this section was to get as close as possible to the Khintchine-type threshold for making progress on the Fundamental Question, we have not expended much effort in possible relaxations. 
We would like to mention an open problem related to this article. It asks about how much the PPC property is violated for a sequence that has not metric PPC.

Problem Under which conditions on $\left(a_{n}\right)_{n}$ is it true that NPPC $\left(\left(a_{n}\right)_{n}\right)$ having full Lebesgue measure implies that the set of $\alpha \in[0,1]$ such that for all $s>0$

$$
\limsup _{N \rightarrow \infty}([-s, s], \alpha, N)=\infty
$$

$$
N \rightarrow \infty
$$

\section{holds also has full Lebesgue measure?}

Acknowledgements Open access funding provided by Austrian Science Fund (FWF). Both authors would like to express their gratitude towards C. Aistleitner for introducing them to the topic of this article, and valuable discussions. Thanks are also due to Thomas Bloom, Sam Chow, Ayla Gafni, and Aled Walker for helpful conversations.

Open Access This article is distributed under the terms of the Creative Commons Attribution 4.0 International License (http://creativecommons.org/licenses/by/4.0/), which permits unrestricted use, distribution, and reproduction in any medium, provided you give appropriate credit to the original author(s) and the source, provide a link to the Creative Commons license, and indicate if changes were made.

\section{References}

1. Aichinger, I., Aistleitner, C.., Larcher, G.: Quasi-energy-spectra, pair correlations, and additive combinatorics. arXiv:1708.08590 (2017)

2. Aistleitner, C., Larcher, G., Lewko, M.: Additive energy and the hausdorff dimension of the exceptional set in metric pair correlation problems. Isr. J. Math. 222(1), 463-485 (2017)

3. Aistleitner, C., Lachmann, T., Pausinger, F.: Pair correlations and equidistribution. J. Number Theory 182, 206-220 (2018)

4. Bloom, T., Chow, S., Gafni, A., Walker, A.: Additive energy and the metric Poissonian property. Mathematika. Preprint available at arXiv:1709.02634 (2017)

5. Boca, F.P., Zaharescu, A.: Pair correlation of values of rational functions (mod p). Duke Math. J. 105(2), 267-307 (2000)

6. Bondarenko, A., Seip, K.: GCD sums and complete sets of square-free numbers. Bull. Lond. Math. Soc. 47(1), 29-41 (2015)

7. Bugeaud, Y.: Approximation by Algebraic Numbers, vol. 160. Cambridge University Press, Cambridge (2004)

8. Harman, G.: Metric Number Theory. London Mathematical Society Monographs. Oxford University Press, Oxford (1998)

9. Heath-Brown, D.R.: Pair correlation for fractional parts of $n^{2}$. Math. Proc. Camb. Philos. Soc. 148(3), 385-407 (2010)

10. Larcher, G., Stockinger, W.: Pair correlation of sequences (\}$a_{n} \alpha\{)_{n \in N}$ with maximal order of additive energy. Preprint available at arXiv:1802.02901 (2018)

11. Larcher, G., Stockinger, W.: Some negative results related to Poissonian pair correlation problems. Preprint available at arXiv:1803.05236 (2018)

12. Larcher, G.: Remark on a result of Bourgain on Poissonian pair correlation. Preprint available at arXiv:1711.08663 (2017)

13. Larcher, G., Grepstad, S.: On pair correlation and discrepancy. Arch. Math. 109(2), 143-149 (2017)

14. Marklof, J., Strömbergsson, A.: Equidistribution of Kronecker sequences along closed horocycles. Geom. Funct. Anal. 13(6), 1239-1280 (2003)

15. Pirsic, Í., Stockinger, W.: The Champernowne constant is not Poissonian. Preprint available at arXiv:1710.09313 (2017)

16. Rudnick, Z., Sarnak, P.: The pair correlation function of fractional parts of polynomials. Comm. Math. Phys. 194(1), 61-70 (1998) 
17. Rudnick, Z., Zaharescu, A.: A metric result on the pair correlation of fractional parts of sequences. Acta Arith. 89(3), 283-293 (1999)

18. Rudnick, Z., Sarnak, P., Zaharescu, A.: The distribution of spacings between the fractional parts of $n^{2}$. Invent. Math. 145(1), 37-57 (2001)

19. Steinerberger, S.: Localized quantitative criteria for equidistribution. Acta. Arith. 180(2), 183-199 (2017)

20. Tao, T., Vu, V.: Additive Combinatorics. Cambridge University Press, Cambridge (2006)

21. Truelsen, J.L.: Divisor problems and the pair correlation for the fractional parts of $n^{2}$. Int. Math. Res. Not. 16, 3144-3183 (2010)

22. Walker, A.: The Primes are not metric Poissonian. Mathematika 64(1), 230-236 (2018)

23. Weyl, H.: Über die Gleichverteilung von Zahlen mod. Eins. Math. Ann. 77(3), 313-352 (1916) 